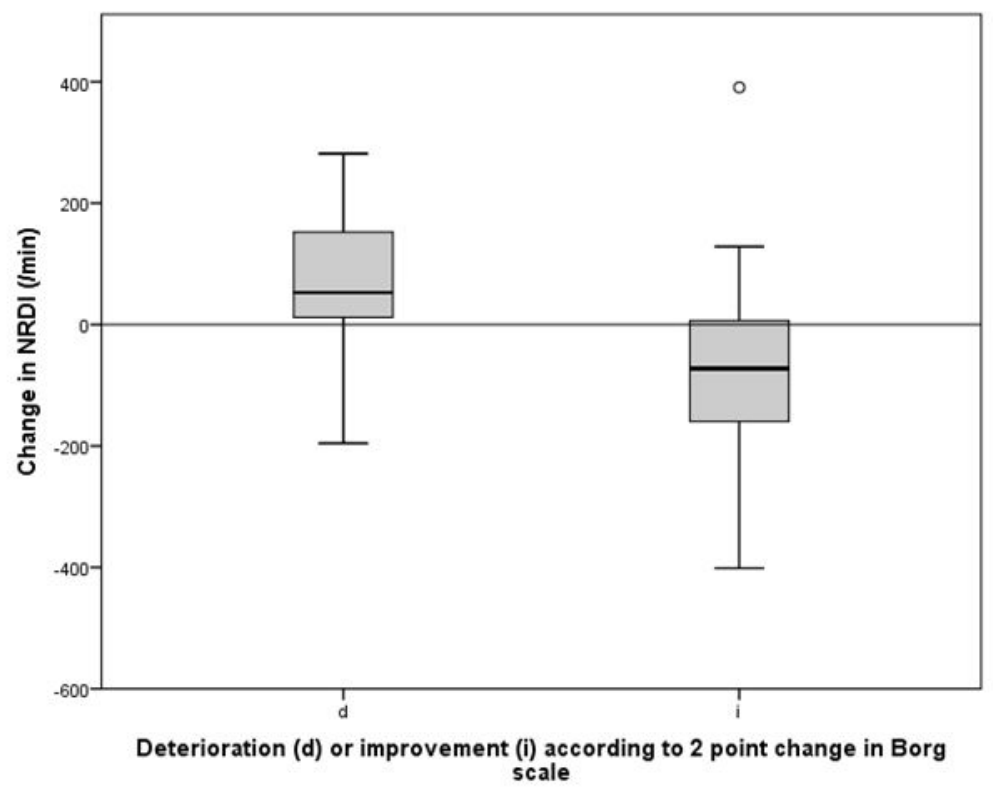

Figure 1 Change in neural respiratory drive index (NRDI) in patients whose Borg scores deteriorated by $\geq 2$ points (d), and those whose Borg scores improved by $\geq 2$ points (i). Independent $t$-test $p<0.001$

Abstract P36 Figure 1

Abstract P37 Table 1

ACCP 1974 (>=15\% baseline)

ATS/ERS 2005 (>12\% baseline and $>200 \mathrm{ml})$

ERS 1994 (>9\% predicted)

ERS 1995 (>10\% predicted)

$>12 \%$ of the baseline

$>400 \mathrm{mls}$

$>200 \mathrm{mls}$

$>12 \%$ of the predicted
M:F

1.04

1.30

0.91

0.93

1.02

2.34

1.49

0.87

${ }^{*} p<0.0001$

ns not significant

Conclusions Neural respiratory drive, as measured by parasternal muscle EMG, is effective in the detection of improvement and deterioration during $\mathrm{AECOPD}$, and demonstrates potential as a physiological biomarker to monitor clinical change.

\title{
P37 WHICH BRONCHODILATOR REVERSIBILITY DEFINITION BEST AVOIDS BIAS
}

doi:10.1136/thoraxjnl-2012-202678.178

${ }^{1} \mathrm{HM}$ Ward, 'BG Cooper, ${ }^{2} \mathrm{MR}$ Miller. ' University Hospitals of Birmingham, Birmingham, United Kingdom; 'Institute of Occupational and Environmental Medicine, University of Birmingham, Birmingham, United Kingdom

Introduction There is little agreement between the international respiratory societies over how bronchodilator reversibility (BDR) should be expressed. Several methods are currently being used worldwide including FEV1 change as \% of predicted, as \% from baseline, as absolute change or a combination of these methods. We explored 8 different definitions of BDR by testing the null hypothesis that the best method for expressing BDR should show no difference between the sexes.

Methods We obtained the pre and post FEV1 results for the first BDR studies using salbutamol $400 \mu \mathrm{g}$ via a spacer for white

Caucasian subjects in our laboratory database up to $11 / 1 / 12$ $[n=5369,49.6 \%$ men, mean (SD) age 50.7 (10.6) years]. The results were expressed as per the definitions of the international societies and also as their individual components if there were combined criteria: $\geq 15 \%$ change from baseline (ACCP 1974), $>12 \%$ change from baseline and >200ml (ATS 1991 and ATS/ERS 2005), >9\% change from predicted (ERS 1994), $>10 \%$ change from predicted (ERS 1995), $>400 \mathrm{ml}$ (BTS), $>12 \%$ baseline, $>200 \mathrm{ml}$, and $>12 \%$ predicted.

Results The distribution of change was right skewed with 416 subjects having a fall in FEV1 post $\mathrm{BD}$. The table shows the median baseline FEV1 and the male to female ratio for those meeting (+ve) and not meeting (-ve) the various BDR criteria. Definitions based on \% change from baseline were biased to positive results in those with smaller pre BD FEV1. BDR criteria including absolute change were biased to those with a larger FEV1 and gave a higher proportion of males with a positive BDR result. The criterion $>400 \mathrm{ml} \mathrm{FEV1} \mathrm{change}$ gave the highest male to female ratio of 2.34. Criteria including both absolute and change as \% predicted had both sex and FEV1 size bias whereas using only change as \% predicted avoided these biases.

Conclusion The most appropriate method for expressing bronchodilator response is based on change as \% of the predicted FEV1 (ERS 1994 or 1995) as it avoids sex and FEV1 size bias. 